\title{
CÂNCER CEREBRAL MODELADO MATEMATICAMENTE
}

\author{
Regiane Aparecida Nunes de Siqueira*, Fernando da Silva Borges*, Kelly Cristiane \\ IAROsZ $^{\dagger}$, Antonio Marcos BAtista ${ }^{\ddagger}$ \\ *Pós Graduação em Ciências \\ Universidade Estadual de Ponta Grossa \\ Ponta Grossa, Paraná, Brasil \\ ${ }^{\dagger}$ Institute for Complex Systems and Mathematical Biology \\ University of Aberdeen \\ Aberdeen, Scotland, United Kingdom \\ † Departamento de Matemática e Estatística \\ Universidade Estadual de Ponta Grossa \\ Ponta Grossa, Paraná, Brasil
}

Emails: ransiqueira@gmail.com, fernandodasilvaborges@gmail.com, kiarosz@gmail.com, antoniomarcosbatista@gmail.com

\begin{abstract}
In this work we analyse mathematical models that describe the dynamics of populations. The models describe tumor cells interaction with immune system and provide a proposed model of control to uncontrolled cell proliferation in brain regions. The proposed immune system consists of resting cells and hunting cells. Resting cells become hunting cells, but not immediately, so there is the insertion of a delay time in differential equations used. Interactions between normal and tumor cells used are competitive for available resources and the chemotherapeutic agent gets into the model as a predator for normal and cancerous cells. In the proposed control of tumor cell proliferation is considered the process of metastasis from a primary to a secondary source with a time delay and it is presented a model that describes brain tumor treatment by injection of chemotherapeutic agents.
\end{abstract}

Keywords - Differential equations with delay, tumors, chemotherapy.

Resumo - Neste trabalho analisa-se modelos matemáticos que descrevem a dinâmica de populações. Os modelos estudados descrevem a interação das células tumorais com o sistema imunológico e propiciam a proposta de um modelo de controle para proliferação celular descontrolada em regiões cerebrais. O sistema imunologico proposto é constituido por células de repouso e celulas de caça. As células de repouso se convertem em células de caça, porém não imediatamente, havendo assim a inserção de um tempo de atraso nas equações diferenciais utilizadas. As interações entre as células normais e tumorais utilizadas são competitivas para os recursos disponiveis e o agente quimioterápico entra no modelo como um predador para células normais e cancerígenas. Na proposta de controle da proliferação de células tumorais considera-se o processo de metastase de um foco primário para um foco secundário com um tempo de atraso e apresenta-se um modelo que descreve o tratamento de um tumor cerebral por meio da injeção de agentes quimioterápicos.

Keywords - Equações diferenciais com atraso, tumores, quimioterapia.

\section{Introdução}

Em organismos de seres vivos tais como anfíbios, peixes, répteis, aves e mamíferos, existem grupos de células ${ }^{1}$ especializadas e organizadas, formando tecidos que compõem órgãos vitais (Otto, 1997). Tais organismos apresentam um crescimento celular cuidadosamente regulado, que corresponde a suas necessidades específicas, porém, é possível um rompimento dos mecanismos reguladores, acarretando um processo de crescimento celular não controlado (Brasil, 2010).

Como exemplo deste descontrole tem-se as neoplasias, comumente chamadas de "tumores". Um tumor pode surgir a partir de alterações genéticas que acabam permitindo que células neoplásicas liberem os mecanismos homeostáticos, que regulam a proliferação celular normal (Hahn

\footnotetext{
${ }^{1}$ Célula é a unidade básica estrutural e funcional dos seres vivos.
}

and Weinberg, 2002).

Neste trabalho apresenta-se um modelo matemático que descreve o desenvolvimento de câncer cerebral. Para tanto, utilizou-se equações diferenciais com atraso, considerando-se interações entre células cerebrais, bem como os efeitos de agentes quimioterápicos (Freedman and Pinho, 2009).

\section{Tumor - Sistema imunológico}

Aqui são apresentadas as soluções de um sistema de equações diferenciais que descrevem o atraso de interação entre as células tumorais e o sistema imunológico. Existem dois ramos de respostas do sistema imunológico: a imunidade humoral e imunidade mediada por células(Banerjee and Sarkar, 2008). Na imunidade humoral a resposta imunológica é realizada por anticorpos, produzi- 
dos pelos linfócitos ${ }^{2} \mathrm{~B}$, diferente da imunidade mediada por células, que são realizadas pelos linfócitos $\mathrm{T}$.

A imunidade mediada por células envolve a produção de linfócitos $\mathrm{T}$ citotóxicos (CTLs), macrófagos ativados, ativação de células $\mathrm{NK}^{3}$ e liberação de várias citocinas em resposta a um antígeno mediado por linfócitos $\mathrm{T}$, essa não envolve quaisquer anticorpos. Os CTLs reconhecem e destroem células infectadas/tumorais. Os linfócitos T citotóxicos ligam-se aos epítopos dos antígenos endógenos ${ }^{4}$ vinculados ao complexo principal de histocompatibilidade (MHC-I) das moléculas da superfície das células infectadas/tumorais e provocam ruptura ou dissolução da membrana plasmática na célula com perforinas (Banerjee and Sarkar, 2008).

É importante considerar duas espécies celulares para modelar a interação tumor-sistema imunológico: células $\mathrm{T}$ e as células tumorais. As células $\mathrm{T}$ podem ser classificadas em células de caça (linfócitos T citotóxicos - CTLs), células T em repouso e células T auxiliares. Os CTLs atacam, destroem ou ingerem as células tumorais. As células $\mathrm{T}$ auxiliares estimulam a liberação de citocinas, que ativam os CTLs para coordenar o contra-ataque. As células $\mathrm{T}$ em repouso podem ser convertidas em CTLs para que assim possam caçar e destruir as células tumorais. Utilizouse o crescimento logístico para modelar o crescimento das células tumorais e células $\mathrm{T}$ em repouso. No modelo, as células tumorais são destruídas em uma taxa proporcional à densidade de células tumorais e células de caça. Além disso, há um decréscimo nas células de caça devido ao encontro com as células tumorais. Considera-se que as células $\mathrm{T}$ em repouso são convertidas em células de caça (CTLs) ou por contato direto com os CTLs ou por contato com citocinas produzidas pelas células $\mathrm{T}$ auxiliares. $\mathrm{O}$ processo de ativação e conversão de células $\mathrm{T}$ em repouso em células $\mathrm{T}$ citotóxicas de caça (CTLs) não é instantâneo, possui um tempo de atraso. Isso faz naturalmente com que o modelo leve em consideração o fator tempo de atraso $(\tau$ em unidades de tempo), no tempo de conversão (de repouso ao estado de caça) e no tempo de crescimento das células de caça. Outra consideração utilizada é que se uma célula foi convertida, nunca retornará para a fase de repouso e de que células ativas morrem em uma probabilidade constante por unidade de tempo. O modelo matemático que descreve tais considerações tem a forma apresentada a seguir

\footnotetext{
${ }^{2}$ Linfócito é um tipo de leucócito (glóbulo branco) presente no sangue.

${ }^{3}$ Células NK (do inglês Natural Killer Cell) ou células exterminadoras naturais são um tipo de linfócitos responsáveis pela defesa específica do organismo.

${ }^{4}$ Endógeno significa que é produzido ou que se desenvolve no interior do organismo.
}

(Banerjee and Sarkar, 2008):

$$
\begin{aligned}
\frac{d M(t)}{d t} & =r_{1} M(t)\left(1-\frac{M(t)}{k_{1}}\right)-\alpha_{1} M(t) N(t), \\
\frac{d N(t)}{d t} & =\beta N(t) Z(t-\tau)-d_{1} N(t) \\
& -\alpha_{2} M(t) N(t), \\
\frac{d Z(t)}{d t} & =r_{2} Z(t)\left(1-\frac{Z(t)}{k_{2}}\right)-\beta N(t) Z(t-\tau),
\end{aligned}
$$

onde $M, N, Z$ são o número de células tumorais, células de caça e células em repouso; $r_{1}$ e $r_{2}>0$ são as taxas de proliferação de células tumorais e células em repouso; $k_{1}, k_{2}>0$ representam, respectivamente, o número máximo de células tumorais e das células em repouso.

O termo $-d_{1} N$ representa a morte natural das células de caça ( $d_{1}$ é uma constante positiva); $-\alpha_{1} M N$ o decrescimo de células tumorais devido ao encontro com as células de caça e $-\alpha_{2} M N$ o decréscimo de células de caça devido ao encontro com as células do tumor. Há um atraso na conversão da fase de repouso para a fase de caça (CTLs), que explica o termo $-\beta N Z(t-\tau)$ na terceira equação. Este atraso na transformação induz um atraso no aumento do número das células de caça, e isso faz com que o termo $\beta N Z(t-\tau)$ esteja presente também na segunda equação.

Na Figura 1 (a) apresenta-se o comportamento do sistema "Tumor - Sistema imunológico" para $\tau=44$, observou-se que para um valores menores de $\tau$ o sistema apresenta poucas oscilações no início do tempo e rapidamente atinge o estado estacionário, conforme a Figura 1 (b) onde utilizou-se $\tau=10$. Já para um $\tau$ maior há muitas oscilações durante todo o tempo mantendo um estado oscilatório, conforme observa-se na Figura 1 (c) para $\tau=46$.

Figura 1: Evolução temporal do número de células do sistema (1) para (a) $\tau=44$, (b) $\tau=10$ e (c) $\tau=46$.
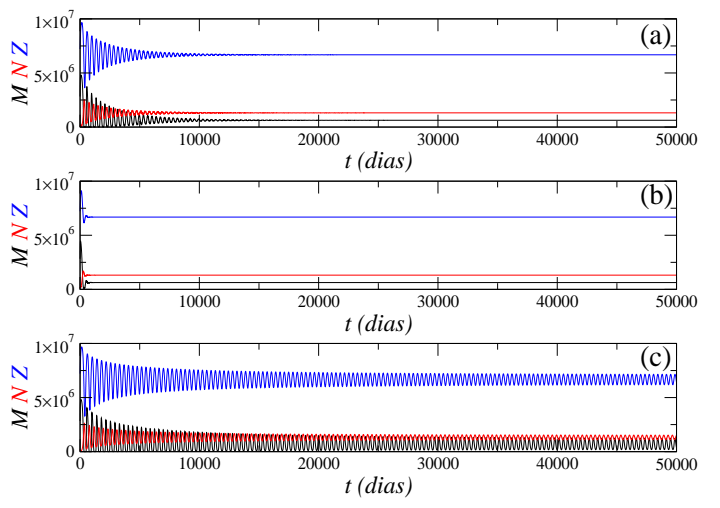

Para valores de atraso a partir de $\tau=45,6 \simeq$ 46 dias, o estado deixa de ser estacionário e passa para um estado oscilatório conforme pode-se observar na Figura 1 (c). 
Para $\tau=45,6$ dias, uma solução de pequena amplitude periódica ocorre e é o caso de bifurcação Hopf. A bifurcação Hopf ocorre quando um ciclo limite é formado em torno de um ponto fixo, o que resulta em soluções estáveis periódicas. Neste modelo a oscilação estável persiste até $\tau=54$. A existência de soluções periódicas é relevante em modelos de tumor. Isto implica que os níveis de tumor podem oscilar em torno de um ponto fixo, mesmo na ausência de qualquer tratamento.

\section{Controle de câncer}

Considera-se um sistema com seis equações diferenciais, três representam as interações entre as células normais, células tumorais, e agentes de quimioterapia no foco primário e as demais equações representam as mesmas interações, no foco secundário. Também é levado em conta a migração das células tumorais a partir do foco primário para o foco secundário com um tempo de atraso (Pinho et al., 2002).

Modelou-se o sistema por meio de cinco equações diferenciais ordinárias e uma equação diferencial funcional. Visa-se simular as interações entre as células normais, as células tumorais e os agentes de quimioterapia em cada foco ${ }^{5}$, com metástase ${ }^{6}$ do tumor do foco primário para o foco secundário.

Sejam $x_{1}(t)$ e $x_{2}(t)$ as concentrações de células normais e tumorais no foco primário, respectivamente, e $y(t)$ a concentração do agente de quimioterapia no foco primário. Da mesma forma, define-se $u_{1}(t)$ equivalente a $x_{1}(t), u_{2}(t)$ equivalente a $x_{2}(t)$ e $z(t)$ equivalente a $y(t)$ no foco secundário. Isso leva ao modelo (Pinho et al., 2002):

$$
\begin{aligned}
\dot{x}_{1}(t) & =\alpha_{1} x_{1}(t)\left(1-\frac{x_{1}(t)}{K_{1}}\right)-q_{1} x_{1}(t) x_{2}(t) \\
& -\frac{p_{1} x_{1}(t) y(t)}{a_{1}+x_{1}(t)} \\
\dot{x}_{2}(t) & =\alpha_{2} x_{2}(t)\left(1-\frac{x_{2}(t)}{K_{2}}\right)-q_{2} x_{1}(t) x_{2}(t) \\
& -\frac{p_{2} x_{2}(t) y(t)}{a_{2}+x_{2}(t)}-\delta x_{2}(t), \\
\dot{y}(t) & =\Delta-\left[\xi+\frac{c_{1} x_{1}(t)}{a_{1}+x_{1}(t)}+\frac{c_{2} x_{2}(t)}{a_{2}+x_{2}(t)}\right] y(t),
\end{aligned}
$$

\footnotetext{
${ }^{5}$ órgão fisiológico infectado pelo tumor.

${ }^{6}$ Processo pelo qual o câncer se espalha a partir de um foco primário num dado órgão para um foco secundário em outro órgão fisiológico por meio da corrente sanguínea ou do sistema linfático.
}

$$
\begin{aligned}
\dot{u}_{1}(t) & =\beta_{1} u_{1}(t)\left(1-\frac{u_{1}(t)}{L_{1}}\right)-r_{1} u_{1}(t) u_{2}(t) \\
& -\frac{s_{1} u_{1}(t) z(t)}{b_{1}+u_{1}(t)}, \\
\dot{u}_{2}(t) & =\beta_{2} u_{2}(t)\left(1-\frac{u_{2}(t)}{L_{2}}\right)-r_{2} u_{1}(t) u_{2}(t) \\
& -\frac{s_{2} u_{2}(t) z(t)}{b_{2}+u_{2}(t)}+\varepsilon \delta x_{2}(t-\tau), \\
\dot{z}(t) & =\Phi-\left[\eta+\frac{d_{1} u_{1}(t)}{b_{1}+u_{1}(t)}+\frac{d_{2} u_{2}(t)}{b_{2}+u_{2}(t)}\right] z(t) .
\end{aligned}
$$

Para as células normais e tumorais em ambos os focos com a concorrência em cada foco entre as células normais e as cancerígenas utilizou-se um crescimento logistico. O agente de quimioterapia age como um predador em ambas as células normais e cancerígenas em cada foco. Além disso, há um possível tempo de atraso $(\tau)$ o qual as células tumorais levam do foco primário até a interação com o foco secundário.

Apresenta-se na Figura 2 a evolução temporal do número de células com $x_{2}=0,5$. Nas Figuras 2 (a) e 2 (b) apresenta-se o comportamento do agente de quimioterapia no foco primário e secundário, respectivamente. Observa-se na Figura 2 (c) a evolução das células normais no primeiro foco do tumor, verificando que com a condição inicial $x_{1}=500$, e quimioterapia $y=90$, rapidamente o sistema dobra o número de células normais, contudo, na sequência vai a zero, representando um estado em que as células tumorais vencem as normais. Pode ser observado na Figura 2 (e) que iniciando com o número de células tumorais, $x_{2}=0,5$, rapidamente atinge-se o limite $x_{2}=K_{2}=2100$, que representa número máximo de células tumorais que o primeiro foco suporta.

Figura 2: Evolução temporal do controle contínuo de câncer considerando $x_{2}=0,5, \tau=1$ e ação nos focos primário e secundário, respectivamente, em (a) e (b) agente quimioterápico; (c) e (d) células normais; (e) e (f) células tumorais.
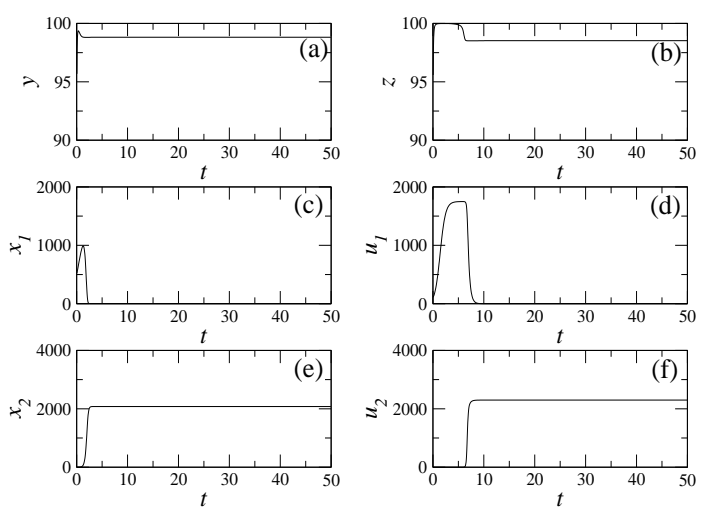

Nas Figuras 2 (d) e 2 (f) observa-se o comportamento das células normais e tumorais, respectivamente, no segundo foco do tumor. É possível verificar um comportamento semelhante ao 
do foco primário, com a condição inicial $u_{1}=100$, rapidamente atinge-se o limite $u_{1}=L_{1}=1750 \mathrm{e}$ na sequência vai a zero, assim como, o número de células tumorais atinge rapidamente o limite $u_{2}=L_{2}=2300$. Dessa forma, na Figura 2, com $x_{2}=0,5$, as soluções aproximam-se do equilíbrio $E_{2}\left(0, \hat{x}_{2}, \hat{y}, 0, \hat{u}_{2}, \hat{z}\right)$, isto é, aproximam-se do equilíbrio de células não normais, ou seja, não há células normais em ambos os focos, representando o estado em que as células tumorais venceram em ambos os focos.

Na Figura 3, utilizando-se as mesmas condições iniciais da Figura 2, contudo o tempo de atraso aumentado para $\tau=10$, observa-se que o efeito de $\tau$ atrasa o momento em que as células tumorais do foco secundário dominam as células normais.

Figura 3: Evolução temporal do controle contínuo de câncer considerando $x_{2}=0,5, \tau=10$ e ação nos focos primário e secundário, respectivamente, em (a) e (b) agente quimioterápico; (c) e (d) células normais; (e) e (f) células tumorais.
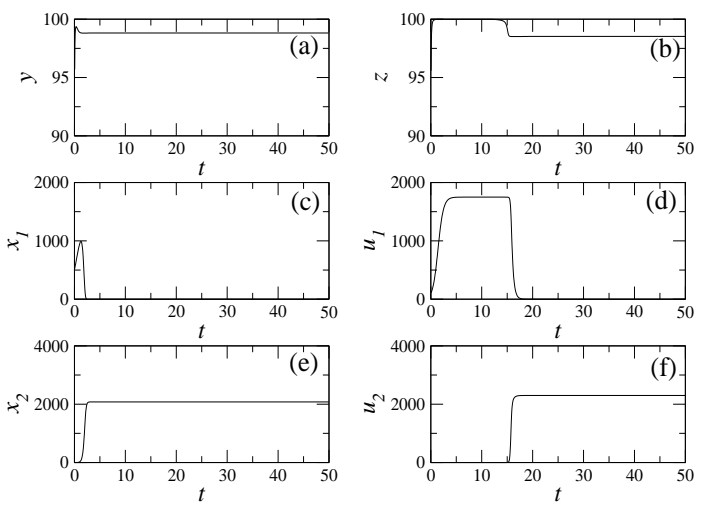

\section{Controle de câncer no cerebral}

Tumores de cérebro são neoplasias raras, representam menos de $2 \%$ de todas as neoplasias malignas, mas apresentam importância epidemiológica devido à sua crescente incidência e elevada morbimortalidade $^{7}$ (Kleihues and Cavenee, 2000). O termo "tumor de cérebro" é bastante geral visto que inclui vários tipos de tumores benignos e malignos, classificados com base na histopatologia em: tumores do tecido neuroepitelial, também conhecidos por neuroglioma ou glioma, incluindo o astrocitoma, astrocitoma anaplásico, glioblastoma, oligodendroglioma e ependimona; tumores das meninges, incluindo meningioma e hemangioblastoma; tumores de células germinativas e tumores da região selar, incluindo tumores da pituitária e craniofaringioma. Os tumores de cérebro mais comuns são os gliomas, que se desenvolvem nas células da glia (Luz, 2010).

\footnotetext{
${ }^{7}$ Morbimortalidade refere-se ao impacto das doenças e das mortes que incorrem em uma sociedade.
}

As células glias dão suporte estrutural e metabólico para os neurônios. Os neurônios não são afetados diretamente pelas células cancerígenas, contudo, o câncer afeta as células glias e não é possível afetar um conjunto de células glias sem afetar de alguma forma os neurônios(Glees, 1955). O aumento da concentração das células cancerígenas acarreta em uma diminuição da concentração de células glias o que produz uma diminuição dos neurônios pela falta de suporte (Lent, 2010). Sendo assim, é necessário considerar o tempo de atraso $\tau$ que as células cancerígenas levam para afetar os neurônios.

Para modelar o crescimento de tumor cerebral considera-se as concentrações de células glias $G(T)$, neuronais $N(T)$ e cancerígenas $C(T)$ e o tratamento do tumor por quimioterapia $Q(T)$. Assim, o modelo, inspirado nos modelos abordados nas seções anteriores e em (Pinho et al., 2013), resulta em:

$$
\begin{aligned}
\frac{d G(T)}{d T} & =\Omega_{1} G(T)\left(1-\frac{G(T)}{K_{1}}\right)-\Psi_{1} G(T) C(T) \\
& -\frac{P_{1} G(T) Q(T)}{A_{1}+G(T)}, \\
\frac{d C(T)}{d T} & =\Omega_{2} C(T)\left(1-\frac{C(T)}{K_{2}}\right)-\Psi_{2} G(T) C(T) \\
& -\frac{P_{2} C(T) Q(T)}{A_{2}+C(T)}, \\
\frac{d N(T)}{d T} & =-\Psi_{3}[G(0)-G(T-\tau)] \delta_{i 0} N(T) \\
\frac{d Q(T)}{d T} & =\frac{P_{3} N(T) Q(T)}{A_{3}+N(T)},
\end{aligned}
$$

onde o delta de Kronecker $\delta_{i j}$ é definido como

$$
\delta_{i j}=\left\{\begin{array}{lll}
1, & \text { se } & i=j, \\
0, & \text { se } & i \neq j,
\end{array}\right.
$$

sendo $i, j \in Z$, para este caso $j=0$ e

$$
\begin{cases}i=1, & \text { se } \quad \dot{G} \geq 0, \\ i=0, & \text { se } \quad \dot{G}<0 .\end{cases}
$$

Considera-se o crescimento logístico para a concentração de células glias e cancerígenas, ocorre um decréscimo no número de células glias devido ao encontro com células cancerígenas, bem como no número de células cancerígenas pelo encontro com células glias, explicando os termos $-\Psi_{1} G(T) C(T)$ e $-\Psi_{2} G(T) C(T)$ nas duas primeiras equações. $\mathrm{O}$ decréscimo de células glias e cancerícenas devido ao efeito da quimioterapia é explicada pelos termos $-P_{1} G(T) Q(T) /\left(A_{1}+G(T)\right)$ e $-P_{2} C(T) Q(T) /\left(A_{2}+C(T)\right)$. Tais termos referem-se ao efeito da quimioterapia, considerando a resposta funcional do tipo MichaelisMenten (Aroesty et al., 1973), sendo a taxa de 
mortalidade per capita das células tumorais dada por $P_{k} Q /\left(A_{k}+Q\right), k=1,2,3$.

Embora existam casos de renovação funcional do neurônio lesado ou a formação de um novo neurônio, este modelo desconsidera tais casos, visto que a formação de um neurônio, denominada neurogênese, é um processo lento e, na maioria das vezes, a nova célula nervosa nasce em determinada região cerebral para depois migrar em direção ao local onde outro neurônio foi destruído. Em muitos casos, as redes de neurônios são rearranjadas e as sinapses (conexões entre eles) são reforçadas por meio de estimulações.

Dessa forma, a equação que descreve a dinâmica da concentração de neurônios deve incluir o termo $-\Psi_{3}[G(0)-G(T-\tau)] N(T)$ que descreve o dinâmica de neurônios em relação as glias. Este termo descreve a perda de neurônios quando existe perda de células glias, isto é, quando $\dot{G}<0$. Se $\dot{G}>0$, significa que não há perda de células glias e neste caso não há perda de neurônios, isto justifica o uso do delta de Kronecker $\delta_{i j}$ que controla o sinal de $\dot{G}$ de forma que, no caso de sinal positivo, fica apenas o termo $-P_{3} N(T) Q(T) /\left(A_{3}+N(T)\right)$ que descreve o efeito da quimioterapia sobre os neurônios.

O termo $P_{k} Q /\left(A_{k}+Q\right), \quad k=1,2,3$ é interpretado como a perda de viabilidade celular por ação de droga, onde $Q$ é a concentração local de quimioterapia; $P_{k}$ e $A_{k}$ podem ser escolhidos de modo a refletir o impacto do ciclo específico da droga. $A_{k}$ segue a partir das observações em que a saturação do efeito do fármaco ocorre para uma certa concentração de droga, e $P_{k}$, resulta da correspondente fração sobrevivente de droga.

A dinâmica de concentração de agentes quimioterápicos é descrita por $\Phi-\zeta Q(T)$ onde $\Phi$ é a taxa de infusão contínua de quimioterapia e $\zeta$ é a taxa de eliminação do agente de quimioterapia.

Figura 4: Evolução temporal do controle contínuo de câncer cerebral, considerando $\Delta=75$ e $\tau=5$. Em (a) glias; (b) neurônios; (c) câncer cerebral e em (d) agente quimioterápico.
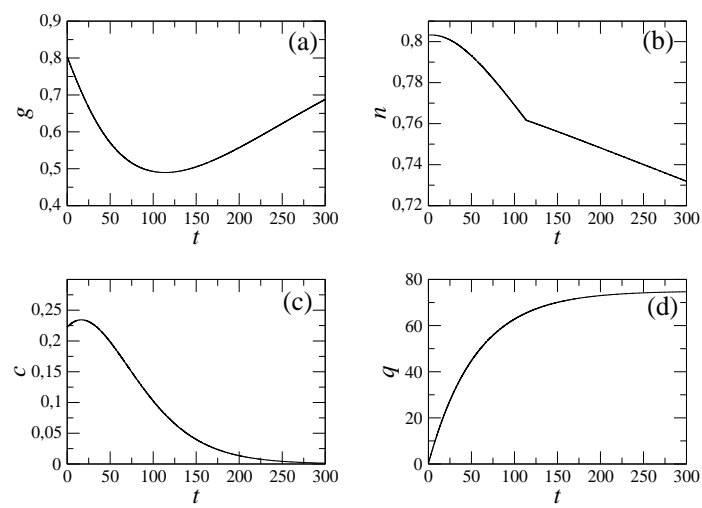

O sistema de controle de câncer cerebral atinge o equilíbrio $E(\bar{g}, 0, \bar{n}, \bar{q})$, que representa um estado de cura, apresentado na Figura 4 (a) glias, (b) neurônios, (c) câncer cerebral e (d) agente quimioterápico.

Figura 5: Evolução temporal do controle contínuo de câncer cerebral, considerando $\Delta=0$ e $\tau=5$. Em (a) glias; (b) neurônios; (c) câncer cerebral e em (d) agente quimioterápico.
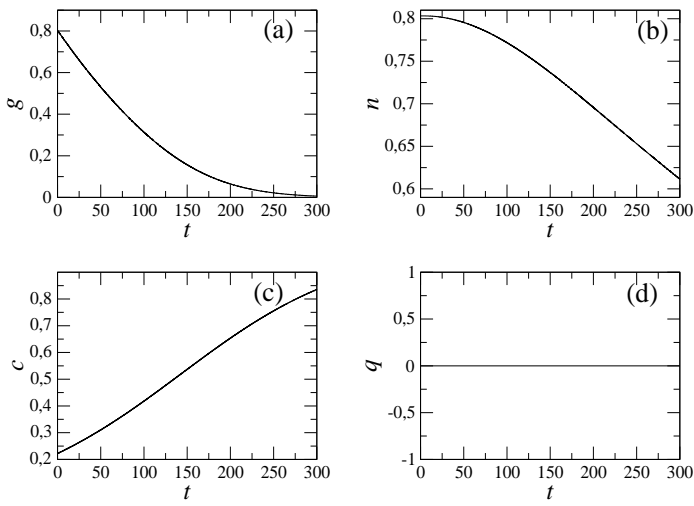

A fim de verificar a "hipótese do câncer"(Pinho et al., 2002), foi eliminado a quimioterapia, fazendo $\Delta=0$ e admitindo-se a condição inicial $q(0)=0$, o sistema atinge o equilíbrio $E_{2}(0, \bar{c}, \bar{n}, 0)$ que representa um estado em que o câncer vence as células glias, apresentado na Figura 5(a) glias, (b) neurônios, (c) câncer cerebral e (d) agente quimioterápico.

\section{Conclusões}

Apresentou-se a análise dos efeitos das interações entre células tumorais e células do sistema imunológico por meio de um sistema de equações diferenciais não-lineares com atraso. O modelo proposto é relativamente simples. A principal diferença entre o sistema apresentado e os outros nesse sentido é o uso da equação diferencial com atraso, que aparece naturalmente quando se consideram as interações celulares.

Analisou-se um modelo de controle do câncer em que ocorre a metástase das células tumorais. Utilizou-seo um sistema de seis equações diferenciais para o modelo. Nas análises, foi incorporouse a "hipótese de câncer", a qual afirma que não havendo o tratamento, o câncer sempre ganha a competição com as células normais em qualquer foco da doença.

Sendo assim, exceto em poucos casos em que as respostas imunológicas naturais são capazes de conter o câncer, as células tumorais acabam por superar as células normais a ponto de causar a morte, independentemente de quando ocorre a metástase, ou seja, independente do valor de $\tau$.

Observou-se que mantendo as mesmas condições iniciais e variando-se apenas o tempo de atraso $\tau$ o modelo atinge o mesmo equilíbrio. Contudo, o efeito de $\tau$ atrasa o momento em que 
as células tumorais do foco secundário dominam as células normais desse foco.

Com relação ao modelo de controle de tumor cerebral, observa-se que no início há um decréscimo de células glias, contudo, quando a doença é estabilizada, as glias recuperam-se atingindo um limite e as células cancerígenas, devido ao efeito da quimioterapia vão rapidamente a zero. Qaunto ao comportamento do número de neurônios, quando há diminuição no número de glias há queda no número de neurônios, contudo, quando as glias recuperam-se, não há recuperação do número de neurônios, continuam a diminir pelo efeito da quimioterapia.

\section{Agradecimentos}

A realização do trabalho foi possível devido ao auxílio financeiro das seguintes agências: $\mathrm{CNPq}$, CAPES e Fundação Araucária.

\section{Referências}

Aroesty, J., Lincoln, T., Shapiro, N. and Boccia, G. (1973). Tumor growth and chemotherapy: Mathematical methods, computer simulations, and experimental foundations, Mathematical Biosciences (17): 243-300. DOI: 10.1016/0025-5564(73)90072-2

Banerjee, S. and Sarkar, R. R. (2008). Delayinduced model for tumor-immune interaction and control of malignant tumor growth, BioSystems (91): 268-288. DOI: 10.1016/j.biosystems.2007.10.002

Brasil (2010). Manual de bases técnicas da oncologia - SIA/SUS - Sistema de Informações Ambulatoriais, SIA.

Freedman, H. I. and Pinho, S. T. R. (2009). Stability criteria for the cure state in a cancer model with radiation treatment, Nonlinear Analysis: Real World Applications (10): 2709-2715. DOI: 10.1016/j.nonrwa.2008.08.001

Glees, P. (1955). Neuroglia: Morphology and Function, Blackwell.

Hahn, W. C. and Weinberg, R. A. (2002). Modelling the molecular circuitry of cancer, $\mathrm{Na}$ ture Reviews (2): 331-341.

Kleihues, P. and Cavenee, W. K. (2000). Pathology and genetics of tumours of the nervous system, IARC Press, Lyon.

Lent, R. (2010). Cem bilhões de neurônios? conceitos fundamentais de neurociência, Atheneu.

Luz, G. F. (2010). Fatores da dieta e tumores de cérebro, Universidade Federal da Bahia, Dissertação de Mestrado.
Otto, S. E. (1997). Emfermagem em oncologia, Lusociência - Edições técnicas e científicas, Ltda.

Pinho, R. T. R., Freedman, H. I. and Nani, F. (2002). A chemotherapy model for the treatment of cancer with metastasis, Mathemati-cal and Computer Modeling (36): 773-803. DOI: 10.1016/S0895-7177(02)00227-3

Pinho, S. T. R., Barcelar, F. S., Andrade, R. F. S. and Freedman, H. I. (2013). A mathematical model for the effect of anti-angiogenic therapy in the treatment of cancer tumours by chemotherapy, Nonlinear Analysis: Real World Applications (14): 815-828. DOI: 10.1016/j.nonrwa.2012.07.034 